\title{
Excited-state quantum phase transitions in spin-orbit-coupled Bose gases
}

\author{
J. Cabedo $\odot$ and A. Celi® \\ Departament de Física, Universitat Autònoma de Barcelona, E-08193 Bellaterra, Spain
}

(Received 27 September 2021; revised 23 November 2021; accepted 26 November 2021; published 27 December 2021)

\begin{abstract}
Excited-state quantum phase transitions depend on and reveal the structure of the whole spectrum of manybody systems. While they are theoretically well understood, finding suitable signatures and detecting them in actual experiments remains challenging. For instance, in spinor gases, excited-state phases have been identified and characterized through a topological order parameter that is challenging to measure in experiments. Here we propose the Raman-dressed spin-orbit-coupled gas as a novel platform to explore excited-state quantum phase transitions. In a weakly coupled regime, the dressed system is equivalent to a spinor gas with tunable spin-spin interactions. Through this equivalence we are able to identify excited-state phases in the Raman-dressed Bose gas. The phases are characterized by the behavior of the spatial density modulations, or stripes, induced by spin-orbit coupling, and can in principle be measured in current state-of-the-art experiments with ultracold atoms. Conversely, we show that the properties of the excited phase can be exploited to prepare stripe states with large and stable density modulations.
\end{abstract}

DOI: 10.1103/PhysRevResearch.3.043215

\section{INTRODUCTION}

Harnessing quantum matter with light is at the heart of quantum technology [1,2]. Artificial spin-orbit coupling (SOC) in ultracold atom gases is a prominent example [3-5]. Spinor gasses dressed by Raman coupling [6,7] interact differently [8], host stripe phases [9,10] with supersolidlike properties (see also [11], for dipolar gases realization see [12-14]), or even realize a topological gauge theory [15]. Here we propose to use Raman-dressed spin-orbit-coupled gases for studying dynamical [16] and excited [17] quantum phase transitions in spinor Bose-Einstein condensates (BECs).

In analogy to ground-state quantum phase transitions $[18,19]$, dynamical and excited-state quantum phase transitions involve the existence of singularities, respectively, in the time evolution and in the energy (or an order parameter) of an excited energy level, and can extend across the excitation spectra. Dynamical phase transitions have been demonstrated in quench experiments with cold atoms in optical lattices [20-22] and cavities [23], trapped ions [24,25], and with superconducting qubits [26]. At the same time, excited-state quantum phase (ESQP) transitions have been shown to occur in a variety of models [27-35], and have been observed in superconducting microwave Dirac billiards [36]. Recently, dynamical and ESQP transitions have been theoretically $[37,38]$ and experimentally $[39,40]$ studied in spin-1 BECs with spin-changing collisions.

Published by the American Physical Society under the terms of the Creative Commons Attribution 4.0 International license. Further distribution of this work must maintain attribution to the author(s) and the published article's title, journal citation, and DOI.
In [41] we showed that the Raman-dressed spin-1 SOC gas at low energy can be understood as an artificial spin-1 gas with tunable spin-changing collisions that can be adjusted with the intensity of the Raman beams. For weak Raman couplings and zero total magnetization, the dressed system is well described by a one-axis-twisting collective spin Hamiltonian [42-44]. The realization of the same model in undressed spinor condensates has led to the observation of various quantum many-body phenomena [45], including the formation of spin domains and topological defects [46-56], and the generation of macroscopic entanglement [57-69], with prospects for metrological applications [70].

The map to pseudospin degrees of freedom (see Fig. 1) highlights the potential advantage of SOC dressed gases for engineering quantum many-body physics: the enhanced tunability of the system and the built-in entanglement between the emerging collective spin structures and the orbital degrees of freedom. In this work we employ these unique features to identify a novel excited-stripe (ES) phase of the spin-1 SOC gas. The phase is in correspondence to the brokenaxisymmetry $\left(\mathrm{BA}^{\prime}\right)$ excited phase of the effective collective spin model, discussed in [38], which is characterized by a topological order parameter, and can extend over the whole spectrum of the Hamiltonian. In the SOC gas, ES phase comprises the classical phase-space trajectories with nonzero time average of the spatial modulations of the density of the gas.

We exploit the relationship between the topological order parameter and the stability of the density modulations in the SOC gas to design a novel detection protocol for the ESQPs of the spinor gas. In the dressed gas, having an interferometer built-in generated by SOC makes a measurement of the contrast of the stripe equivalent to a simultaneous measurement of the amplitude and phase of the dressed spin components. Remarkably, this approach benefits from an intrinsic robustness to magnetic fluctuations, which constrains the current 


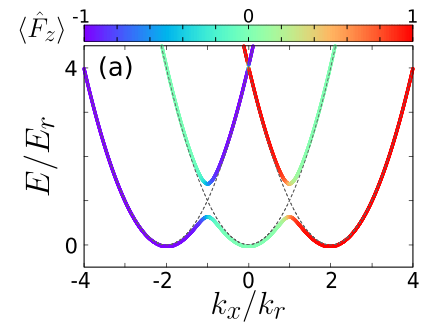

(b)
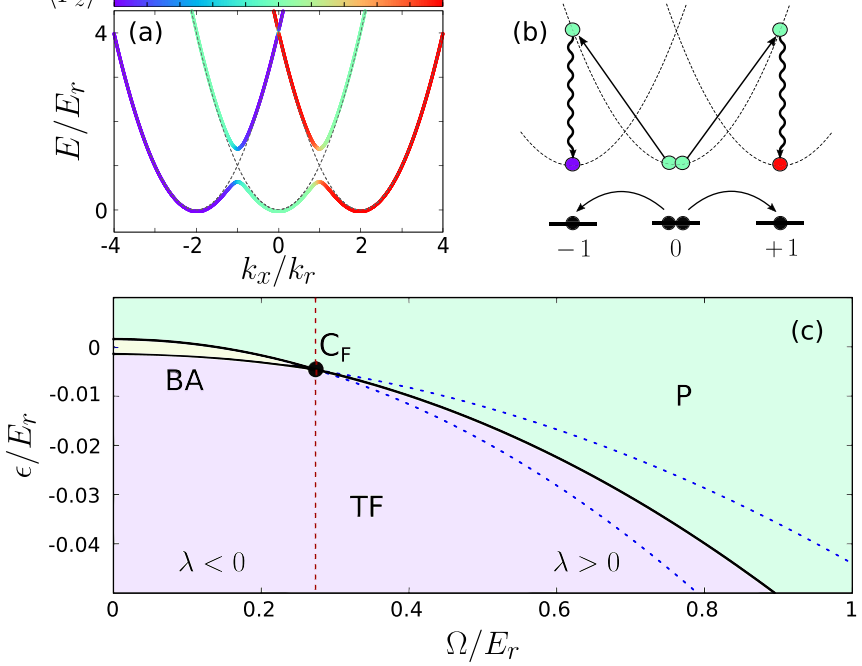

FIG. 1. Pseudospin dynamics in SOC BECs. (a) Dispersion bands of Hamiltonian (1), setting $\Omega=0.75 E_{r}, \delta=0$, and $\epsilon=$ $\Omega^{2} / 16 E_{r}$. The corresponding mean value of $\hat{F}_{z}$ for the band states is indicated with the color texture. The undressed bands are shown in dashed gray. (b) Schematic representation of an effective spin-changing collision process enabled by Raman transitions (represented in wavy lines). (c) Phase diagram of the dressed spin Hamiltonian (3) as a function of the Raman Rabi frequency $\Omega$ and effective quadratic Zeeman shift $\epsilon$, for $\bar{n}=7.5 \times 10^{13} \mathrm{~cm}^{-3}$ and using the values of $a_{0}$ and $a_{2}$ for ${ }^{87} \mathrm{Rb}$ given in [45]. The polar $(\mathrm{P})$, twin-Fock (TF), and broken-axisymmetry (BA) phases meet at the tricritical point $C_{F}$ (black dot). The dashed red vertical line at $\Omega_{c}=4 E_{r} \sqrt{\left|g_{a}\right| / g_{s}}$ separates the ferromagnetic $(\lambda<0)$ and the antiferromagnetic $(\lambda>0)$ regimes of the effective Hamiltonian. The blue dotted lines enclose the region of parameters around the P-TF transition where the $\mathrm{BA}^{\prime}$ excited-state quantum phase takes place (see Sec. III), with its boundaries located at $\tilde{\epsilon}= \pm 2 \lambda$ in the thermodynamic limit.

proposals for detecting the excited phases of the model in spinor gases with intrinsic spin changing collisions [38].

Finally, through the effective model we are able to provide a robust protocol to prepare striped states. The ES phase of the gas can be accessed from an initially unpolarized gas via crossing an ESQP transition in a two-step quench scheme. With such an approach, we show that the ES phase can be realized in current state-of-the-art experiments with spin-1 SOC gases, with the prepared states exhibiting large and stable density modulations. At the same time, the proposal introduces a novel procedure to access the striped regime of the spin-1 with SOC, which as a ground-state phase has a very narrow region of stability [71] and it has yet to be experimentally demonstrated.

The paper is organized as follows. In Sec. II we briefly review the Raman-dressed spin-1 gas and its description as a collective pseudospin Hamiltonian with tunable spin interactions. In Sec. III we introduce the novel ES phase of the dressed condensate, and show that its experimental signature can provide a new means to detect the ESQP transitions of the collective spin model. In Sec. IV we propose a robust protocol to prepare ES states, which we benchmark in Sec. V. Finally, we briefly recap and draw our conclusions in Sec. VI.

\section{RAMAN-DRESSED GAS AS AN ARTIFICIAL SPINOR GAS}

We consider a spin-1 BEC of $N$ atoms of mass $m$ subject to synthetic SOC with equal Rashba and Dresselhaus contributions, as experimentally realized via Raman-coupling two Zeeman pairs $\{|1,1\rangle,|1,0\rangle\}$ and $\{|1,0\rangle,|1,-1\rangle\}$ independently, as in [72]. In the presence of dressing, the kinetic Hamiltonian can be written as

$$
\hat{\mathcal{H}}_{\mathrm{k}}=\frac{\hbar^{2}}{2 m}\left(\boldsymbol{k}-2 k_{r} \hat{F}_{z} \boldsymbol{e}_{x}\right)^{2}+\frac{\Omega}{\sqrt{2}} \hat{F}_{x}+\delta \hat{F}_{z}+\epsilon \hat{F}_{z}^{2},
$$

where $\hbar \hat{F}_{j}$ are the spin-1 matrices. Here $\Omega$ quantifies the Raman coupling strength. By simultaneously adjusting the detuning from the resonance of each Raman pair, the strengths of an effective quadrupole tensor field and a magnetic field term $\epsilon$ and $\delta$, respectively, can be independently tuned in the laboratory (see Methods from [72]).

The many-body scenario for the Raman dressed gas in mean-field regime is captured by the energy functional

$$
\begin{aligned}
E[\boldsymbol{\psi}]= & \int d \boldsymbol{r}\left[\boldsymbol{\psi}^{*}\left(\hat{\mathcal{H}}_{\mathrm{k}}+V_{\mathrm{t}}\right) \boldsymbol{\psi}+\frac{g_{s}}{2}\left|\boldsymbol{\psi}^{4}\right|\right. \\
& \left.+\frac{g_{a}}{2} \sum_{j}\left(\boldsymbol{\psi}^{*} \hat{F}_{j} \boldsymbol{\psi}\right)^{2}\right],
\end{aligned}
$$

where $\psi=\left(\psi_{-1}, \psi_{0}, \psi_{1}\right)^{T}$ is the spinor condensate wave function, normalized to the total number of particles as $\int d \boldsymbol{r} \boldsymbol{\psi}^{\dagger} \boldsymbol{\psi}=N$. The spin-symmetric and nonsymmetric interaction couplings are given by $g_{s}=4 \pi \hbar^{2}\left(a_{0}+2 a_{2}\right) / 3 m$ and $g_{a}=4 \pi \hbar^{2}\left(a_{2}-a_{0}\right) / 3 m$, where $a_{0}$ and $a_{2}$ are the scattering lengths in the $F=0$ and $F=2$ channels, respectively. For simplicity we will consider that the gas is confined with an isotropic harmonic potential $V_{\mathrm{t}}=\frac{1}{2} m \omega_{\mathrm{t}}^{2} \boldsymbol{r}^{2}$.

In this work we focus on the weak Raman coupling regime, where $\Omega$ is smaller than the Raman single-photon recoil energy $E_{r}$. We label the recoil momentum as $\hbar k_{r}$, so that $E_{r}=\frac{\hbar^{2} k_{r}^{2}}{2 m}$. Furthermore, we will consider $E_{r} \gg \delta, \epsilon$. In this regime, the lowest dispersion band of $\hat{\mathcal{H}}_{\mathrm{k}}$ has three different minima $\boldsymbol{k}_{j} \sim 2 j k_{r} \boldsymbol{e}_{z}$, with $j \in\{-1,0,1\}$, as illustrated in Fig. 1(a). As shown in [41], in these conditions the dynamics of the dressed gas is equivalent to the one of an effective spinor gas with Raman-mediated spin-changing collisions [see Fig. 1(b)]. For small condensates, the low-energy landscape of the weakly coupled gas can be restricted to just three self-consistent modes, and the system is then well described by a collective pseudospin Hamiltonian. We label the collective pseudospin operators as $\hat{L}_{x, y, z}=\sum_{\mu \nu} \hat{b}_{\mu}^{\dagger}\left(\hat{F}_{x, y, z}\right)_{\mu \nu} \hat{b}_{\nu}$ and $\hat{L}_{z z}=\sum_{\mu \nu} \hat{b}_{\mu}^{\dagger}\left(\hat{F}_{z}^{2}\right)_{\mu \nu} \hat{b}_{\nu}$, where the bosonic operators $\hat{b}_{-1}^{\dagger}, \hat{b}_{0}^{\dagger}$, and $\hat{b}_{1}^{\dagger}$ create a particle in the left, middle, and right well mode, respectively. With this notation we restrict ourselves to the zero "magnetization" subspace, where $\hat{L}_{z}=0$, the Hamiltonian reads

$$
\hat{H}=\lambda \frac{\hat{L}^{2}}{2 N}+\tilde{\epsilon} \hat{L}_{z z}
$$


Here $\lambda=\left(g_{a}+g_{s} \frac{\Omega^{2}}{16 E^{2}}\right) \bar{n}$, where $\bar{n}$ is the mean density of the gas. The coefficient $\tilde{\epsilon}$ includes a perturbative correction to $\epsilon$, with $\tilde{\epsilon}=\epsilon+\frac{\Omega^{2}}{16 E_{r}}$.

Hamiltonian (3) is completely equivalent to the one describing the nonlinear coherent spin dynamics of spin-1 BECs where $g_{a} \ll g_{s}$ [43]. Note that such equivalence does not generally extend throughout the Hilbert space, where an additional term proportional to $\hat{L}_{z}^{2}$ breaks the $\mathrm{SO}(3)$ symmetry of the interacting Hamiltonian (see [41]). This fact motivates our restriction to the subspace with zero magnetization. Nonetheless, it should be noted that, as long as the spread in $\hat{L}_{z}$ is much smaller than $N$, the analogy can be straightforwardly extended to subspaces of any magnetization. The phase diagram of Hamiltonian (3) in the $\Omega-\epsilon$ plane is shown in Fig. 1(c). The dashed vertical line at $\Omega=4 E_{r} \sqrt{\left|g_{a}\right| / g_{s}}$ separates the ferromagnetic $(\lambda<0)$ and the antiferromagnetic $(\lambda>0)$ regimes of the dressed-spin dynamics. The antiferromagnetic regime includes the polar $(\mathrm{P})$ phase at $\tilde{\epsilon}(\Omega)>0$, in which all the atoms occupy the middle well mode, and the twin-Fock (TF) phase for $\tilde{\epsilon}(\Omega)<0$, where the atoms evenly occupy both edge well states. The scenario is richer in the ferromagnetic regime, where the effective spin interactions favor the formation of a nonvanishing transverse magnetization. When the effective interaction dominates, this results in the spontaneous breaking of the $\mathrm{SO}(2)$ symmetry of the system [47], giving rise to the so-called broken-axisymmetry (BA) phase [73] in between the $\mathrm{P}$ and TF phases.

\section{ESQPS IN SOC GASES}

Ferromagnetic spin-1 BECs, which are described by Hamiltonian (3) with $\lambda<0$, exhibit ESQP transitions [38], between three separate ESQPs that extend from the ground state phases and span across the whole energy spectrum. The ESQP diagram of (3) in the $\tilde{\epsilon}-\mathcal{E}$ plane is shown in Fig. 2(a) for $\lambda<0$, where $\mathcal{E}=\langle\hat{H}\rangle /(|\lambda| N)$ is the scaled energy per particle of the eigenstates of $\hat{H}$ and $\mathcal{E}_{g}$ is the one of the ground state. The phases $\mathrm{P}^{\prime}, \mathrm{BA}^{\prime}$, and $\mathrm{TF}^{\prime}$ are labeled according to the corresponding ground state phase. On the boundaries between the phases, the mean-field limit of the density of states diverges, as it is expected for an ESQP transition [17]. The boundaries are found at $\mathcal{E}^{*}=\tilde{\epsilon} /|\lambda|$ for $-2<\tilde{\epsilon} /|\lambda|<0$, and at $\mathcal{E}^{*}=0$ for $0<\tilde{\epsilon} /|\lambda|<2$. Notice that, since $\hat{H}(\lambda, \tilde{\epsilon})=$ $-\hat{H}(-\lambda,-\tilde{\epsilon})$, the same three phases also occur for antiferromagnetic gases, but with their boundaries redefined, as shown in Fig. 2(b), with $\mathcal{E}^{*}=0$ for $-2<\tilde{\epsilon} /|\lambda|<0$, and $\mathcal{E}^{*}=\tilde{\epsilon} /|\lambda|$ for $0<\tilde{\epsilon} /|\lambda|<2$.

As discussed in [38], within these ESQPs the classical phase-space trajectories of coherent states can be classified with respect to a topological order parameter (for a similar behavior in the Rabi model, see [74]). Here we show that this order parameter is directly related to the stability of the density modulations in the spin-orbit-coupled gas. We exploit this relationship to provide a novel detection protocol for the ESQPs of the spinor gas.

As in [38], we consider now the set of coherent states $|N, \boldsymbol{n}, \boldsymbol{\theta}\rangle=\frac{1}{\sqrt{N !}}\left(\sum_{j} \sqrt{n_{j}} e^{i \theta_{j}} b_{j}^{\dagger}\right)^{N}|0\rangle$ in the zero magnetization subspace, with $\sum_{j} n_{j}=1$ and $n_{1}=n_{-1}$. In the mean-

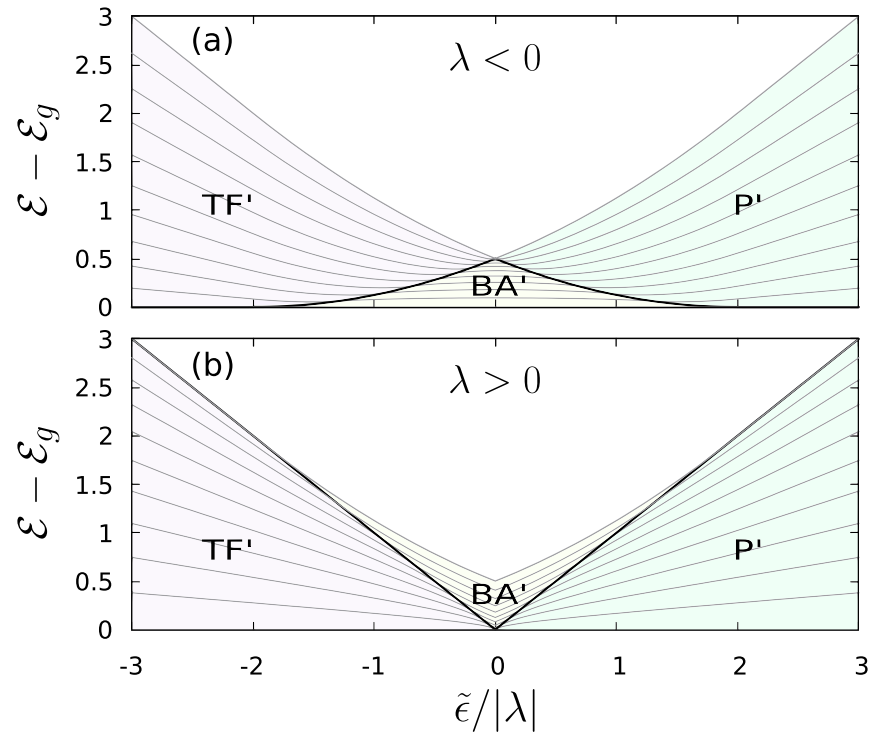

FIG. 2. Excited-state quantum phases (ESQPs) in SOC BECs. ESQP diagram of Hamiltonian (3), which describes the low-energy landscape of spin-1 gases with SOC, for both effective ferromagnetic (a) and antiferromagnetic (b) dressed-spin interactions. The thin gray lines show every 25th eigenvalue of the Hamiltonian for $N=500$. The thick black line indicates the phase boundary at $\mathcal{E}^{*}(\tilde{\epsilon})$.

field limit of (3), the scaled energy per particle is given by

$$
\begin{aligned}
\mathcal{E}(\boldsymbol{n}, \boldsymbol{\theta}) & =\langle N, \boldsymbol{n}, \boldsymbol{\theta}|\hat{H}| N, \boldsymbol{n}, \boldsymbol{\theta}\rangle /|\lambda| N \\
& =\operatorname{sgn}(\lambda) 2\left(1-n_{0}\right) n_{0} \cos ^{2} \theta+\frac{\tilde{\epsilon}}{|\lambda|}\left(1-n_{0}\right),
\end{aligned}
$$

where $\theta=\theta_{0}-\frac{\theta_{1}+\theta_{-1}}{2}$. The corresponding mean-field equations of motion read

$$
\dot{n}_{0}=\frac{|\lambda|}{\hbar} \frac{\partial \mathcal{E}}{\partial \theta}, \quad \dot{\theta}=-\frac{|\lambda|}{\hbar} \frac{\partial \mathcal{E}}{\partial n_{0}} .
$$

The solutions of Eqs. (5) are periodic, and the relationship between the periodicity of $n_{0}(t)$ and $\theta(t)$ varies between the different ESQPs. In the $\mathrm{BA}^{\prime}$ phase, for each point in the $\tilde{\epsilon}-\mathcal{E}$ plane there exist two solutions with disconnected trajectories. In these solutions both $n(t)$ and $\theta(t)$ have the same periodicity. Furthermore, the values that $\theta(t)$ can take are bounded, with $-\pi / 2<\theta(t)<\pi / 2$ in one solution and $\pi / 2<\theta(t)<3 \pi / 2$ in the other. Conversely, in the $\mathrm{P}^{\prime}$ and $\mathrm{TF}^{\prime}$ phases the solution is unique at each point. Labeling the periodicity in $n(t)$ by $\tau$, in the $\mathrm{P}^{\prime}$ and $\mathrm{TF}^{\prime}$ phases of the ferromagnetic diagram one has $\theta(t+\tau)=\theta(t) \pm \pi$ (see $[38,75,76]$ for more details). In [38] they introduce the winding number

$$
w=\frac{1}{\pi}[\theta(\tau)-\theta(0)],
$$

which can be interpreted as a topological order parameter that distinguishes between the three excited phases. It takes the value $w=-1,0,1$ for any mean-field trajectory within the $\mathrm{P}^{\prime}, \mathrm{BA}^{\prime}$, and $\mathrm{TF}^{\prime}$ phases, respectively. In the antiferromagnetic diagram, the sign of $w$ is flipped with respect to the ferromagnetic case. 


\section{A. The excited-stripe phase}

Remarkably we can relate the phase space trajectories $\left[n_{0}(t), \theta(t)\right]$ that coherent pseudospin states follow to the properties of the Raman-dressed atomic cloud. We can write the mean-field wave function of the gas as $\boldsymbol{\psi}(\boldsymbol{r})=$ $\sqrt{N} \sum_{j} \sqrt{n}_{j} \boldsymbol{\phi}_{j}(\boldsymbol{r}) e^{i \theta_{j}}$, where we label the three self-consistent modes around $\boldsymbol{k}_{j}$ as $\boldsymbol{\phi}_{j}$. As the three modes are tightly located at the vicinity of the respective band minima $\boldsymbol{k}_{j}$, we can approximate them by plane waves times a slowly varying envelope function, which for simplicity we omit in the following. Then, up to second order in $\Omega / 8 E_{r}$, and neglecting the corrections $\propto(\epsilon+\delta) \Omega^{2} / E_{r}^{3}$, we can write

$$
\begin{gathered}
\boldsymbol{\phi}_{\mathbf{1}}(\boldsymbol{r}) \propto e^{i k_{1} x}\left[1-\frac{1}{2}\left(\frac{\Omega}{8 E_{r}}\right)^{2}, \frac{\Omega}{8 E_{r}}, 0\right]^{T}, \\
\boldsymbol{\phi}_{\mathbf{0}}(\boldsymbol{r}) \propto e^{i k_{0} x}\left[\frac{\Omega}{8 E_{r}}, 1-\left(\frac{\Omega}{8 E_{r}}\right)^{2}, \frac{\Omega}{8 E_{r}}\right]^{T}, \\
\boldsymbol{\phi}_{-\mathbf{1}}(\boldsymbol{r}) \propto e^{i k_{-1} x}\left[0, \frac{\Omega}{8 E_{r}}, 1-\frac{1}{2}\left(\frac{\Omega}{8 E_{r}}\right)^{2}\right]^{T} .
\end{gathered}
$$

At $\delta=0, \boldsymbol{k}_{0}=0$, and $\boldsymbol{k}_{1}=-\boldsymbol{k}_{-1}$. In these conditions, the spatial density of the gas reads

$$
n(\boldsymbol{r}, t) \sim \bar{n}\left(1+\frac{\Delta n(x, t)}{\bar{n}}\right),
$$

where

$$
\begin{aligned}
\Delta n(x, t)= & \bar{n} \cos \left(k_{1} x-\Delta\right) \frac{\Omega}{E_{r}} \sqrt{\frac{n_{0}(t)\left[1-n_{0}(t)\right]}{2}} \cos \theta(t) \\
& +O\left[\left(\Omega / 8 E_{r}\right)^{2}\right] .
\end{aligned}
$$

Here $\Delta=\theta_{1}-\theta_{-1}$ is the phase difference between the modes at the edge minima, which is a constant of motion at $\delta=0$. In this way, the mean-field solutions of (3) exhibit spatial density modulations that depend both on $n_{0}$ and $\theta$, with a relative amplitude given by

$$
A(t)=\frac{\Omega}{E_{r}} \sqrt{\frac{n_{0}(t)\left[1-n_{0}(t)\right]}{2}} \cos \theta(t) .
$$

Let us evaluate the behavior of these density modulations in the different phases. In both the $\mathrm{P}^{\prime}$ and $\mathrm{TF}^{\prime}$ excited phases, $n_{0}(t+\tau)=n(t)$ and $\cos \theta(t+\tau)=-\cos \theta(t)$. It follows that

$$
\frac{1}{2 \tau} \int_{0}^{2 \tau} d t A(t)=0
$$

and so

$$
\lim _{T \rightarrow \infty} \frac{1}{T} \int_{0}^{T} d t A(t)=0,
$$

for all solutions in the $\mathrm{P}^{\prime}$ and $\mathrm{TF}^{\prime}$ phases. Thus, while an excited state in such phases can exhibit spatial density modulations at a given time, such modulations vanish in the time-averaged density profile.

The situation is different for the $\mathrm{BA}^{\prime}$ phase. There, for each $\tilde{\epsilon}$ and $\mathcal{E}$, one solution fulfills $\cos \theta(t)>0$ for all $t$ while in the other $\cos \theta(t)<0$, and thus

$$
\lim _{T \rightarrow \infty}\left|\frac{1}{T} \int_{0}^{T} d t A(t)\right|>0 .
$$

Therefore, we can define a new observable that distinguishes a novel ESQP of the SOC spin-1 gas, which we label as excitedstripe phase (ES). The classical solutions exhibit a nonzero time-averaged amplitude of the spatial density modulations, or stripes, in the region of parameters that corresponds to the $\mathrm{BA}^{\prime}$ ESQP of the effective dressed spin model of (3). The topological order parameter $w$ therein is then associated with the stability of the stripes in the Raman dressed spin-1 gas. This stability is well understood from the locking of the relative spinor phase $\theta$ in the classical mean-field trajectories when $w=0$, which arises from the effective dressed spin-changing collisions in the gas.

Notice that in presence of a nonzero detuning $\delta$, the phase of the modulations $\Delta$ [see Eq. (9)] becomes time dependent, with $\dot{\Delta}=\dot{\theta}_{1}-\dot{\theta}_{-1}=2 \delta / \hbar$. While the amplitude of the stripes remains unchanged at leading order, this time dependence of the phase results into vanishing modulations in the timeaveraged density profile in the laboratory frame, regardless of the behavior of $A(t)$. However, there always exist a frame comoving with the modulation where time averaging of modulations yields the same nonzero value as at $\delta=0$. In practice, the ES phase can be easily distinguished in the presence of nonzero detuning, or even time dependent, from the behavior of the contrast of the modulations over time, as discussed in detailed in Sec. III C.

In the ES phase, the contrast of the stripes increases with $\Omega$, and, thus, is larger in the antiferromagnetic regime of (3), where $\Omega>\Omega_{c}$. At the same time, for nearly spin-symmetric gases such as ${ }^{87} \mathrm{Rb}$, the region of parameters where the ES can exist is much broader there [indicated with blue-dotted lines in Fig. 1(c)]. Yet in this regime the stripe phase does not occur in the ground state of the Raman dressed gas, and one may suspect the gas to undergo a phase separation between the different spin components over time. Still, within the validity of three-mode truncation that leads to (3), phase separation does not occur, and thus the effective model predicts that the stripe phase will persists as excited states even at $\Omega>\Omega_{c}$ (see [41]).

In the next section we assess by comparison with the mean-field evolution of the whole gas the validity of such a truncation, which is equivalent to the single-spatial mode approximation in undressed antiferromagnetic spinor condensates. As the latter, it holds better the smaller the condensate and for zero total magnetization [77]. As for the latter, it is notoriously difficult to determine analytically its precise range of validity. Naturally the physical requirement on the Hamiltonian of the gas for the single-spatial mode approximation to hold is that its nonsymmetric part has to be a perturbation of the symmetric part, so that $\lambda \ll g_{s} \bar{n}$ and $\lambda \ll \hbar \omega_{t}$.

\section{B. The ES phase: Gross-Pitaevskii results}

To verify the predictions of model (3) for Raman dressed SOC gases, we simulate the Gross-Pitaevskii equation (GPE) 

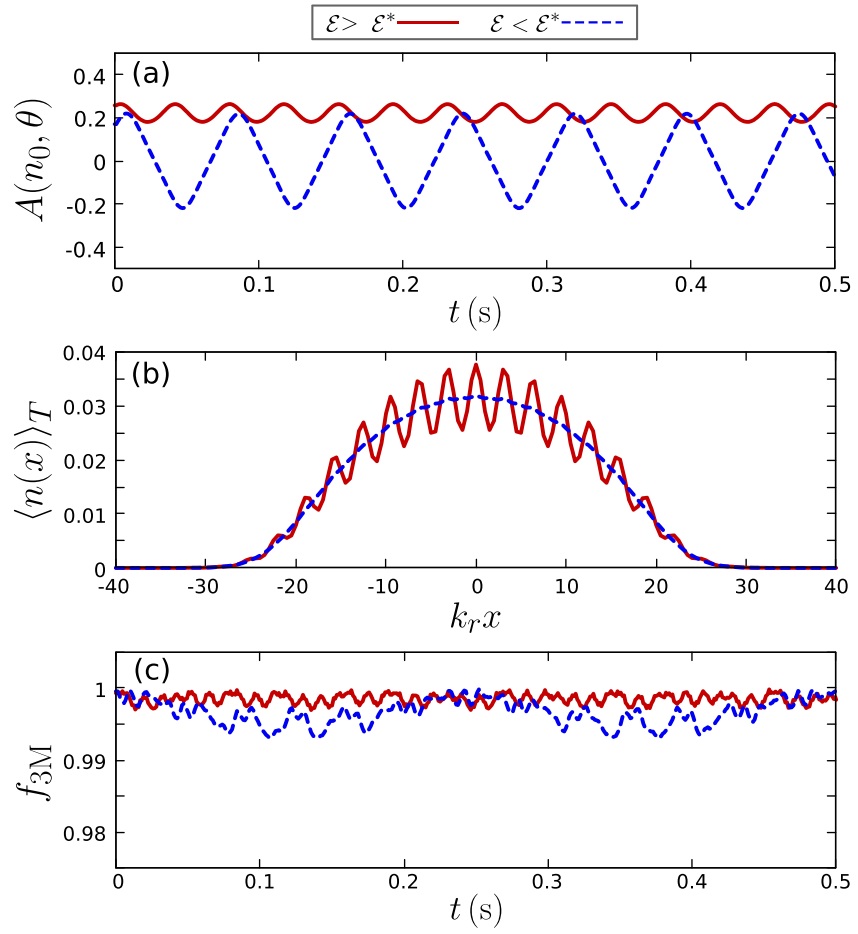

FIG. 3. Signature of the excited-state stripe phase. (a) Relative amplitude $A(t)$ of the spatial modulations for a dressed condensate of $N=10^{4}$ particles prepared with $\Omega=0.75 E_{r}, \omega_{t}=2 \pi \times 140 \mathrm{~Hz}$, $\delta=0$, and $\tilde{\epsilon}=-0.5|\lambda|$, computed using the GPE (14). Solid red: $A(t)$ for a state initially at $n_{0}(0)=0.5$ and $\theta=0.1 \pi$, with $\mathcal{E}>\mathcal{E}^{*}$ (ES phase). Dashed blue: $A(t)$ for an initial state at $n_{0}(0)=0.5$ and $\theta=0.3 \pi$, with $\mathcal{E}<\mathcal{E}^{*}$ ( $\mathrm{T}^{\prime}$ phase). (b) Corresponding time-averaged density profile of the condensate $\langle n(x)\rangle_{T}$ averaged over $T=0.5 \mathrm{~s}$. When $\mathcal{E}>\mathcal{E}^{*}$, the spatial modulation in $\langle n(x)\rangle_{T}$ does not vanish with increasing $T$. (c) Fraction of the condensate that remains within the subspace spanned by the self-consistent modes $\phi_{j}$.

of the whole system:

$$
i \hbar \dot{\psi}_{j}=\delta E[\boldsymbol{\psi}] / \delta \psi_{j}^{*},
$$

where $E[\boldsymbol{\psi}]$ is the energy functional in (2). We calculate the self-consistent modes $\boldsymbol{\phi}_{j}$ via imaginary time evolution of the GPE. The modes can be easily obtained by projecting the ground state at $\delta=\tilde{\epsilon}=0$, which populates the three well states, into the well separated regions around each minimum of the lowest band. We then define $n_{0}=b_{0}^{*} b_{0}$ and $\theta=\arg \left(b_{0}\right)-\left[\arg \left(b_{1}\right)+\arg \left(b_{-1}\right)\right] / 2$, with

$$
b_{j}=\frac{1}{N} \int d \boldsymbol{r} \phi_{j}^{*}(\boldsymbol{r}) \cdot \boldsymbol{\psi}(\boldsymbol{r}) .
$$

We consider small ${ }^{87} \mathrm{Rb}$ condensates in the $F=1$ hyperfine manifold, with $E_{r} / \hbar=2 \pi \times 3678 \mathrm{~Hz}, k_{r}=7.95 \times 10^{6} \mathrm{~m}^{-1}$ [72]. We use the corresponding values $a_{0}=101.8 a_{B}$ and $a_{2}=$ $100.4 a_{B}$ for the scattering lengths in the different channels, taken from [45], where $a_{B}$ is the Bohr radius.

In Fig. 3(a) we plot the relative amplitude $A(t)$ as a function of time for two different states prepared at $\Omega=0.75 E_{r}$, $\omega_{t}=2 \pi \times 140 \mathrm{~Hz}$, and $\delta=0$ with $N=10^{4}$. In both cases we adjust $\epsilon$ so that $\tilde{\epsilon}=-0.5|\lambda|$ and set $n_{0}(0)=0.5$. We then evolve the initial state with the GPE (14). In one trajectory (in solid red), the state is initialized at $\theta=0.1 \pi$, with a corresponding $\mathcal{E}>\mathcal{E}^{*}=0$, and thus expected to be in the ES phase. Indeed, in agreement with the effective model, $A(t)$ is periodic and remains positive (or negative) at any time $t$, due to the spinor phase being bounded along the mean-field trajectory. Conversely, the dashed blue line corresponds to a trajectory with $\theta(0)=0.3 \pi$, and so $\mathcal{E}<\mathcal{E}^{*}$, thus out of the ES phase [see Fig. 2(b)]. In this case the amplitude oscillates between positive and negative values, averaging to 0 over a period. In Fig. 3(b) we show the corresponding time-averaged density profile of the condensate, given by

$$
\langle n(x)\rangle_{T}=\frac{1}{T} \int_{t_{0}}^{t_{0}+T} d t \int d y d z|\boldsymbol{\psi}(\boldsymbol{r})|^{2},
$$

and averaged over a time $T=500 \mathrm{~ms}$. As expected, $\langle n\rangle_{T}$ exhibits large modulations when $\mathcal{E}>\mathcal{E}^{*}=0$, while these vanish for $\mathcal{E}<\mathcal{E}^{*}=0$. In Fig. 3(c) we plot the fraction of atoms that remain within the three-mode subspace, or fidelity $f_{3 M}=$ $\frac{1}{N^{2}} \sum_{j}\left|\int d \boldsymbol{r} \boldsymbol{\phi}_{j}^{*} \cdot \boldsymbol{\psi}\right|^{2}$, as a function of time, which highlights the accuracy of the approximation in this regime of parameters.

As exemplified by the results shown in Fig. 3, the GPE analysis of the Raman dressed gas supports the predictions of the dressed spin model in a broad, and experimentally accessible, range of parameters. We stress that the stripe phase as an excited-state quantum phase is only well defined and understood within the three-mode subspace, where the robustness of the spatial density modulations is enabled by the collective spin structure of the effective Hamiltonian. The contrast of the modulations in $\langle n(x)\rangle_{T}$ is very sensitive on the degree of accuracy of the truncation, which in turn depends both on the strength of the effective spin interaction coefficient $|\lambda|$ and on the total number of particles.

This sensitivity is illustrated in Fig. 4, where we show the values of the time-averaged amplitude $\langle A\rangle_{T}=\frac{1}{T} \int_{0}^{T} d t A(t)$ and fidelity $\left\langle f_{3 \mathrm{M}}\right\rangle_{T}=\frac{1}{T} \int_{0}^{T} d t f_{3 \mathrm{M}}(t)$ for a state initialized at $n_{0}=0.5$ and $\theta=0.1 \pi$ and evolved under Eq. (14), for several values of $\Omega$ in Fig. 4(a), and for a varying total number of particles in Fig. 4(b). In all cases, $\omega_{\mathrm{t}}$ is adjusted so that $\bar{n}=7.5 \times 10^{13} \mathrm{~cm}^{-3}$, and the quantities are averaged over a total time $T=500 \mathrm{~ms}$. In Fig. 4(a) we set $N=10^{4}$, and in Fig. 4(b) $\Omega=0.75 E_{r}$. While, according to the effective model (3), the state is prepared within the $\mathrm{BA}^{\prime}$ phase, with $\mathcal{E}>\mathcal{E}^{*}$, the contrast of the time-averaged density modulations rapidly vanishes as soon as the fidelity of the three-mode truncation degrades. This is exemplified in Fig. 4(c), where we plot the time-averaged density profile for the corresponding trajectories with $N=2 \times 10^{4}$ and $N=4 \times 10^{4}$ from Fig. 4(b). In the latter case, the stripes are absent in the time-averaged density profile, despite having considered the same Raman dressing parameters and atom density than in the former.

It is clear, then, that the collective spin structure is fundamental to the nature of the ES phase. Still, we are able to identify a wide range of parameters for which the few-mode description is accurate, and the behavior of the dressed gas understood in these terms. Furthermore, the direct connection between the ES phase of the Raman dressed gas and the BA' phase of the effective spin model can provide a powerful tool for the detection of the ESQPs of the spinor gas. 

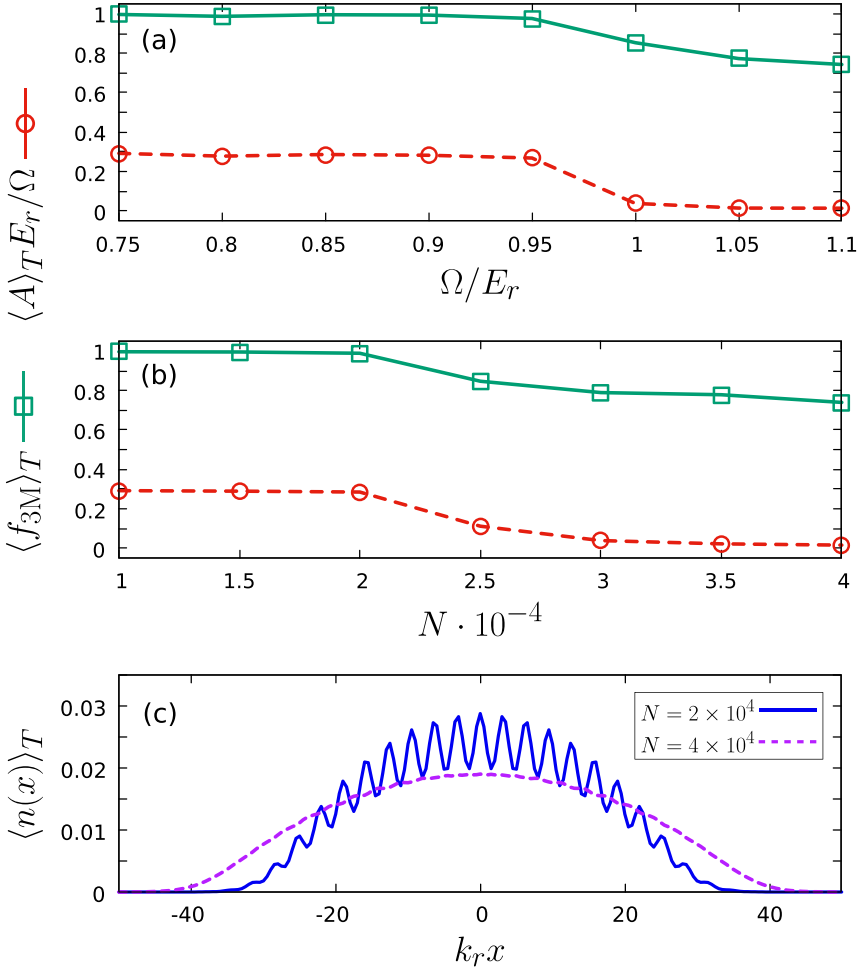

FIG. 4. Robustness of the ES phase. (a) Time-averaged relative amplitude $\langle A\rangle_{T}$ (red circles) and fidelity of the three-mode truncation $\left\langle f_{3 \mathrm{M}}\right\rangle_{T}$ (green squares) as a function of $\Omega$ for a dressed condensate of $N=10^{4}$ particles. The state is prepared with $n_{0}(0)=0.5$ and $\theta=$ $0.1 \pi$, and evolved using (14) with $\tilde{\epsilon}=-0.5|\lambda|$. (b) $\langle A\rangle_{T}$ and $\left\langle f_{3 \mathrm{M}}\right\rangle_{T}$ as a function of $N$ for a state prepared at $n_{0}(0)=0.5$ and $\theta=0.1 \pi$, with $\Omega=0.75 E_{r}$ and $\tilde{\epsilon}=-0.5|\lambda|$. (c) Time-averaged density profile for the corresponding trajectories with $N=2 \times 10^{4}$ (blue solid line) and $N=4 \times 10^{4}$ (purple dashed line) from (b). In all cases, the state is evolved for $T=500 \mathrm{~ms}$ and $\omega_{\mathrm{t}}$ is adjusted to have $\bar{n}=7.5 \times 10^{13}$ $\mathrm{cm}^{-3}$.

\section{Signature of the BA' ESQP}

In [38] the authors propose an experimental scheme to detect the BA' ESQP of a spinor gas. The protocol relies on an interferometric scheme to measure the absolute value of the winding number of (6), $|w|$, where the spins are coupled via an internal-state beam splitter after the state is evolved for a period $T$. Such a scheme faces a major difficulty: the visibility of the projected measurement is very sensitive to the accumulated phase difference between the \pm 1 modes, and hence, to the magnetic field fluctuations in the experiment.

We now show that the realization of the same effective Hamiltonian in the Raman-dressed spinor gas can in principle avoid such a drawback. As discussed in Sec. III A, the amplitude of the spatial density modulations in the dressed gas does not depend at first order in $\Omega / E_{r}$ on the relative phase $\Delta$, and so neither does the contrast or visibility of the modulations, given by $V=2|A|=\left(\Omega / E_{r}\right) \sqrt{2 n_{0}\left(1-n_{0}\right)}|\cos \theta|$. We conveniently define the scaled contrast $\tilde{V}$ as

$$
\tilde{V}=V E_{r} / \Omega=\sqrt{2 n_{0}\left(1-n_{0}\right)}|\cos \theta| .
$$

The measurement of the contrast of the stripes involves, therefore, a simultaneous measurement of the population $n_{0}$ and
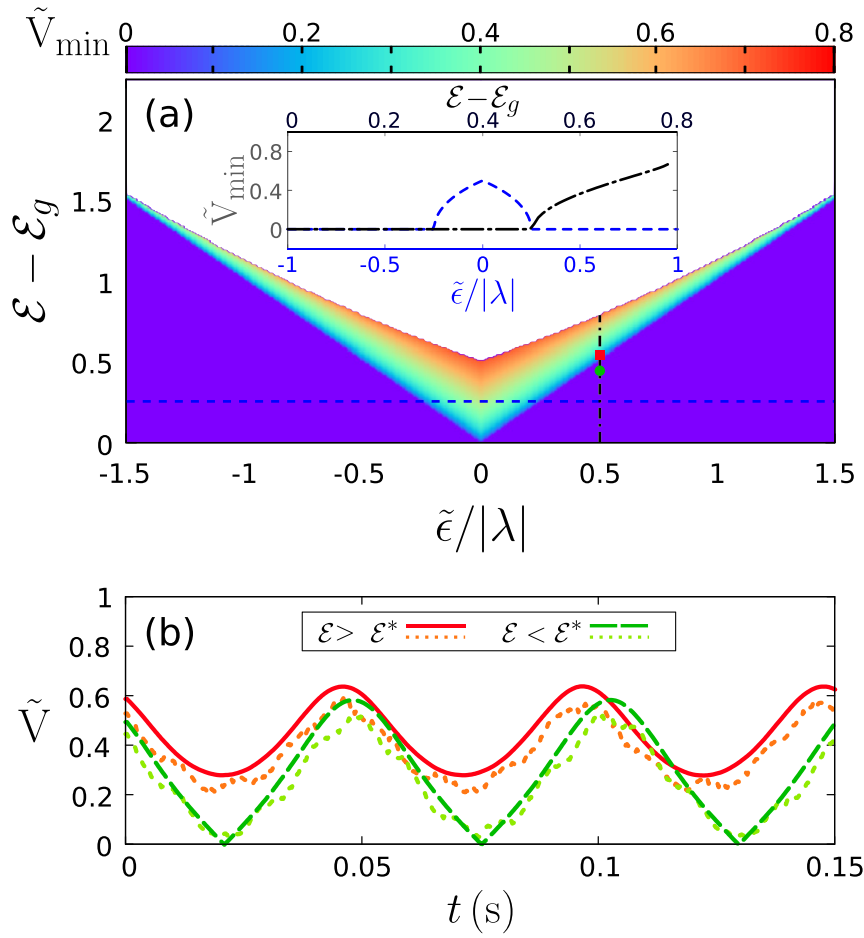

FIG. 5. Stripe contrast as signature of the BA' ESQP. (a) Minimum value $\tilde{V}_{\min }$ of the scaled contrast $\tilde{V}=\sqrt{2 n_{0}\left(1-n_{0}\right)}|\cos \theta|$ along the classical trajectories given by Eqs. (5), as a function of $\tilde{\epsilon}$ and $\mathcal{E}$, computed using Eq. (18). The inset shows $\tilde{V}$ for constant $\mathcal{E}-\mathcal{E}_{g}=0.25$ (dashed-blue line) and $\tilde{\epsilon} /|\lambda|=0.5$ (dashed-dotted black). (b) $\tilde{V}$ as a function of time for two classical trajectories at $\tilde{\epsilon} /|\lambda|=0.5$, in and out of the $\mathrm{BA}^{\prime}(\mathrm{ES})$ phase, indicated in (a) by the red and green square dots, respectively. In solid red, $n_{0}(0)=0.6$ and $\phi(0)=0.174$, with $\mathcal{E}>\mathcal{E}^{*}$. In dashed green, $n_{0}(0)=0.6$ and $\phi(0)=0.243$, with $\mathcal{E}<\mathcal{E}^{*}$. The corresponding values for the peakto-valley scaled contrast of the solutions of the GPE (14) are shown in dotted lines. The values are obtained for a condensate of $N=10^{4}$ and $\bar{n}=7.5 \times 10^{13} \mathrm{~cm}^{-3}$, setting $\Omega=0.75 E_{r}$.

the phase $\theta$. From the behavior of the contrast alone, we can infer the absolute value of the winding number of (6), $|w|$, and, thus, detect the BA' phase of the pseudospin gas- the ES phase of the dressed gas-regardless of the values taken by $\Delta(t)$.

The contrast $\tilde{V}$ is a positive semidefinite quantity and for generic $n_{0}$ can reach zero only when $\theta$ reaches $(2 k+1) \pi / 2$, with $k \in \mathbb{Z}$. This obviously occurs in the $\mathrm{P}^{\prime}$ and $\mathrm{TF}^{\prime}$ phases, where $\theta$ is unbounded, but never occurs in the $\mathrm{BA}^{\prime}$ phase where $|\theta| \leqslant \theta_{\max }<\pi / 2$. Thus, the minimum value $\tilde{V}_{\min }$ of the scaled contrast (17) is a proxy of $|w|$ as it is nonzero only in the BA' phase, as illustrated in Fig. 5(a) where we plot $\tilde{V}_{\text {min }}$ along the classical trajectories as a function of $\tilde{\epsilon}$ and $\mathcal{E}$. The onset of $\tilde{V}_{\min }$ is found at $\mathcal{E}^{*}$ [see the inset in Fig. 5(a)]. In Fig. 5(b) we plot $\tilde{V}$ as a function of time along two trajectories at $\tilde{\epsilon} /|\lambda|=0.5$. We choose the parameters to have one trajectory within the $\mathrm{BA}^{\prime}$ phase, with $\mathcal{E}$ slightly above $\mathcal{E}^{*}$, and the other in the $\mathrm{TF}^{\prime}$ phase, with $\mathcal{E}<\mathcal{E}^{*}$. Finally, in dotted lines we plot the corresponding results from the GPE equation of the dressed and trapped gas (14). The contrast is computed from the relative peak-to-valley difference at the central peak of the 
condensate wave function. We note that values of the minimal contrast shown Fig. 5(a) are obtained analytically using (17). By taking the time derivative of expression (17) and using (5), it is clear that, in the $\mathrm{BA}^{\prime}$ phase, $\tilde{V}$ can only be minimal (or maximal) at $\theta=0$. We then use (4) and (17) with $\theta=0$ to retrieve the analytical expression for $\tilde{V}_{\min }(\mathcal{E}, \tilde{\epsilon})$, which reads

$$
\tilde{V}_{\min }=\sqrt{\mathcal{E}-\frac{\frac{\tilde{\epsilon}}{\lambda}\left(\frac{\tilde{\epsilon}}{\lambda}+2\right)+\frac{|\tilde{\epsilon}|}{\lambda} \sqrt{\left(\frac{\tilde{\epsilon}}{\lambda}-2\right)^{2}-8\left(\mathcal{E}-\frac{\tilde{\epsilon}}{\lambda}\right)}}{4} .}
$$

Such a derivation, however, assumes that the condensates are perfectly located at the three minima of the dispersion band. The presence of trapping leads to a momentum spread of the wave packets, decreasing the actual contrast of the stripes in the cloud. This can be observed in Fig. 5(b), where the peakto-valley contrast evaluated in the condensate wave function is slightly lower than the value predicted by Eq. (17). Nonetheless, for relatively small trapping frequencies, the behavior of the gas in the distinct ESQPs is qualitatively well described by Eq. (17).

In this way we have shown that the realization of the collective spin Hamiltonian (3) with a Raman-dressed artificial spinor gas can provide an alternative approach to the detection of the ESQP transition therein. In the dressed system we propose to exploit the built-in interferometer that arises from Raman dressing, where the three quasimomentum-shifted dressed states can spatially interfere due to their nonzero spin overlap. The behavior of the density modulations arising from such interference signals the value of the topological order parameter that characterizes the $\mathrm{BA}^{\prime}$ phase of the effective spin system introduced in [38]. Our proposal, thus, does not rely on any external interferometric measurement, which results in an intrinsic robustness to magnetic fluctuations. In such a scheme, the precision to delimit the boundary of the $\mathrm{BA}^{\prime}$ phase is subject to the experimental sensitivity associated with the measurements of the density modulations. Remarkably, $\tilde{V}_{\text {min }}$ increases abruptly at the boundary, and the modulations of the ES states remain large at any time of the trajectory even for states close to the transition. This can be understood from the fact that in the classical limit $\tilde{V}_{\text {min }}$ plays the role of the order parameter of a second order phase transition. From (18) we can see that its susceptibility diverges as

$$
\frac{\partial \tilde{V}}{\partial \mathcal{E}} \simeq \frac{\sqrt{C}}{2}(\mathcal{E}-\mathcal{E} *)^{-1 / 2},
$$

where $C=1+\frac{|\epsilon / \lambda|}{2-|\epsilon / \lambda|}$.

At the same time, the properties of the stripe phase as an excited-state phase can be exploited to facilitate the accessibility of stripe states in experiments with spinor gases. In the next section we describe a robust protocol to prepare ES states in a spin-1 spinor gas.

\section{QUENCH EXCITATION OF ES STATES VIA COHERENT SPIN MIXING}

Hamiltonian (3) gives a simple framework to understand the collective behavior of SOC condensates. We now use the predictions of the model to propose a protocol that allows a robust and fast preparation of ES states. By comparing the
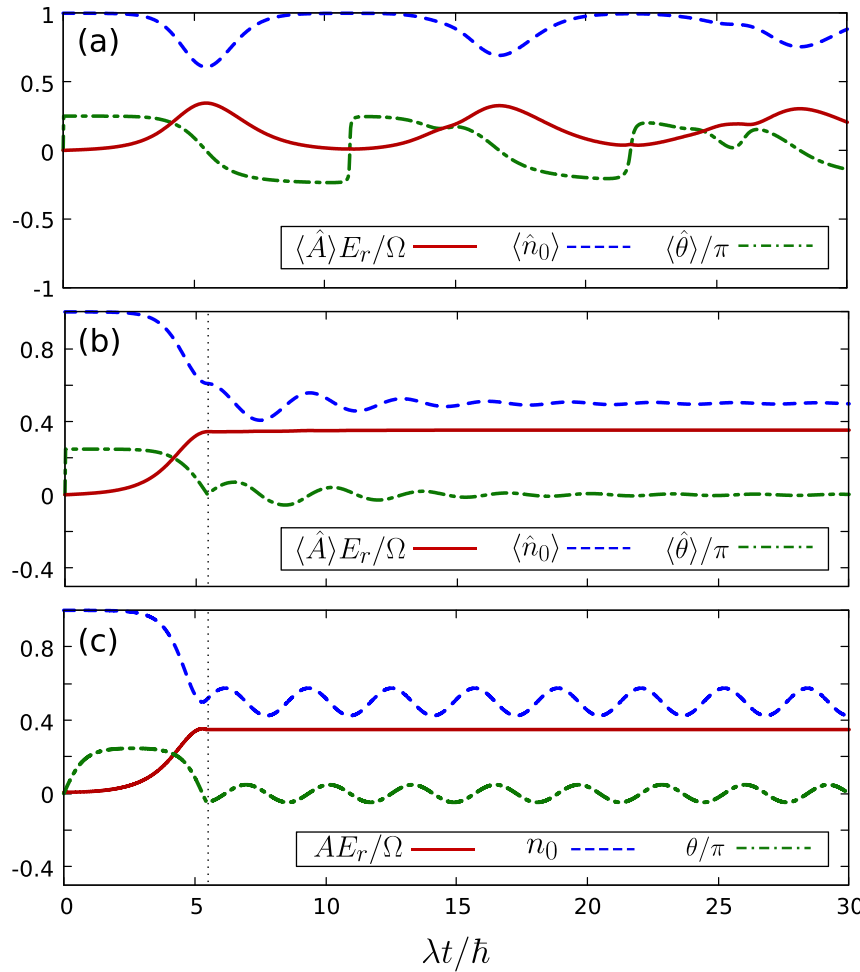

FIG. 6. Excitation of ES states via coherent spin mixing: fewmode predictions. (a) Expected value of $\hat{n}_{0}$ (dashed blue), $\hat{\theta}$ (dashed-dotted green), and $\hat{A}$ (solid red) as a function of time for a state prepared at $t=0$ in $|\psi\rangle(t=0)=\left(\hat{b}_{0}^{\dagger}\right)^{N}|0\rangle$, and evolved under Hamiltonian (3), setting $\tilde{\epsilon} / \lambda=-1$ and $\lambda>0$. (b) The same initial state is evolved with $\tilde{\epsilon} / \lambda=-1$ for a time $t_{1}=5.5 \hbar / \lambda$ (indicated with the vertical dotted gray line), where $\tilde{\epsilon}$ is quenched to 0 . Following the quench, $\langle\hat{A}\rangle$ stabilizes near its maximum value. (c) Classical trajectories for the state $n_{0}(0)=0.9998$ and $\theta(0)=0$ evolved under Eqs. (5), setting $\tilde{\epsilon} / \lambda=-1$ for $t \leqslant t_{1}=5.5 \hbar / \lambda$, and $\tilde{\epsilon}=0$ for $t>t_{1}$.

rescaled contrast (17) and classical energy (5), we notice that $\tilde{V}^{2}=\operatorname{sgn}(\lambda)\left[\mathcal{E}-\tilde{\epsilon} /|\lambda|\left(1-n_{0}\right)\right]$. It immediately follows that at $\tilde{\epsilon}=0, \tilde{V}$ becomes a constant of motion of the classical trajectories as it is proportional to the square root of the classical energy. With this in mind, we propose a two-step quench scheme to access ES states that exhibit large and stable density modulations.

\section{A. Two-step quench scheme: Few-mode predictions}

We consider that the system is initially in the fully polarized state with $n_{0}=1, \theta=0$, where all the atoms occupy the middle well mode. Experimentally, this scenario is very convenient: we can prepare such an state from an undressed polarized condensate in the $m_{f}=0$ spin state simply by adiabatically turning up $\Omega$ while keeping $\tilde{\epsilon}>2|\lambda|$. The preparation is followed by a first quench in $\tilde{\epsilon}$ into the range $-2<\tilde{\epsilon} / \lambda<0$. According to the classical equations of motion (5), such a polarized state is a stationary point of the Hamiltonian at all values of $\tilde{\epsilon}$. However, quantum fluctuations start a coherent spin-mixing dynamics that breaks the stationarity of the state $[37,78,79]$. In Fig. 6(a) we show the expected values of the relative occupation of the middle well mode $\hat{n}_{0}=\frac{1}{N} \hat{b}_{0}^{\dagger} \hat{b}_{0}$, the spinor phase $\hat{\theta}=\frac{1}{2} \arg \left(\hat{b}_{1}^{\dagger} \hat{b}_{-1}^{\dagger} \hat{b}_{0} \hat{b}_{0}\right)$, 
and the relative amplitude $\hat{A}=\left(\Omega / E_{r}\right) \sqrt{\hat{n}_{0}\left(1-\hat{n}_{0}\right) / 2} \cos (\hat{\theta})$ as a function of time, for the initial state $\left(\hat{b}_{0}^{\dagger}\right)^{N}|0\rangle$ evolved under Hamiltonian (3) with $\tilde{\epsilon} / \lambda=-1$ and $\lambda>0$. After some time, $\langle\hat{A}\rangle$ reaches a local maximum. For a coherent state, performing a second quench to $\tilde{\epsilon}=0$ when the maximum is reached would leave $\langle\hat{A}\rangle$ locked at this value. Naturally the quantum trajectories of (3) for noncoherent states and away from the thermodynamic limit may depart from the classical predictions. Nonetheless, as expected, we numerically find a qualitative agreement between classical and quantum trajectories, as shown in Fig. 6(b). In the figure, the initial state $\left(\hat{b}_{0}^{\dagger}\right)^{N}|0\rangle$ is evolved under Hamiltonian (3) with $\tilde{\epsilon}=-\lambda$ for a time $t_{1}=5.5 \hbar / \lambda$, where the Hamiltonian is quenched to $\tilde{\epsilon}=$ 0 . Following the second quench, the relative amplitude $\langle A(t)\rangle$ is rapidly stabilized very near its maximum value $\frac{1}{2 \sqrt{2}} \Omega / E_{r}$. For comparison, in Fig. 6(c) we show the trajectories obtained using Eqs. (5). The state is initially in a coherent state with a very small fraction of atoms in the edge well states, to avoid the classical stationary point at $n_{0}=1$.

\section{B. Excitation of ES states: Gross-Pitaevskii results}

Again we assess further the validity of the scheme with the GPE of the Raman dressed gas. In order to obtain wide and stable density modulations, we take relatively large values of $\Omega$, and consider small condensates to be safely in the three-mode approximation. Figure 7 shows a simulation of the protocol with a condensate of $N=10^{4}$ particles, $\bar{n}=$ $7.5 \times 10^{13} \mathrm{~cm}^{-3}$ and $\Omega=0.75 E_{r}$. In Fig. 6(a) we plot $n_{0}, \theta$, and $A(t)$ as a function of time for a state initially prepared at $n_{0}=0.9998$ and time evolved with the GPE. The state is evolved with $\tilde{\epsilon} / \lambda=-1$ for a time $t_{1}=5.5 \hbar / \lambda$, where $\tilde{\epsilon}$ is quenched to 0 . As expected, $A(t)$ is stabilized after the quench, despite that $n_{0}$ and $\theta$ keep oscillating with time. With the contrast stabilized, the time-averaged density profile exhibits very large density modulations, with over $40 \%$ contrast of the stripes, as shown in Fig. 7(b). In Fig. 7(c) we plot the values of $f_{3 \mathrm{M}}$ during the evolution, which remains very close to 1 for the chosen parameters.

With the two-step quench scheme described, a state with near-maximal density modulations (at a given value of $\Omega$ ) can be reached in a robust and fast manner. In the example shown in Fig. $7, \lambda / \hbar \simeq 2 \pi \times 17.9 \mathrm{~Hz}$, many times larger than the intrinsic spin-mixing rate in a ${ }^{87} \mathrm{Rb}$ undressed gas. The peak in $A(t)$ is reached in about $50 \mathrm{~ms}$. However, the feasibility of the scheme in an actual experiment is subject to the stability of the parameters of the GPE. Several sources of noise can be detrimental to the stability and contrast of the stripes prepared, most notably the fluctuations in the Zeeman levels due to magnetic-field fluctuations and the calibration uncertainty in the intensity of the Raman beams. We briefly discuss these aspects in the next section.

\section{EXPERIMENTAL CONSIDERATIONS}

To benchmark the robustness of the protocol described in Sec. IV, we include fluctuating and randomized parameters in the simulations of the GPE. To account for atom loss, we continuously renormalize the condensate wave function to $N(t)=N(0) \exp (-\gamma t)$, with $\gamma=3.33 \mathrm{~s}^{-1}$, which is com-
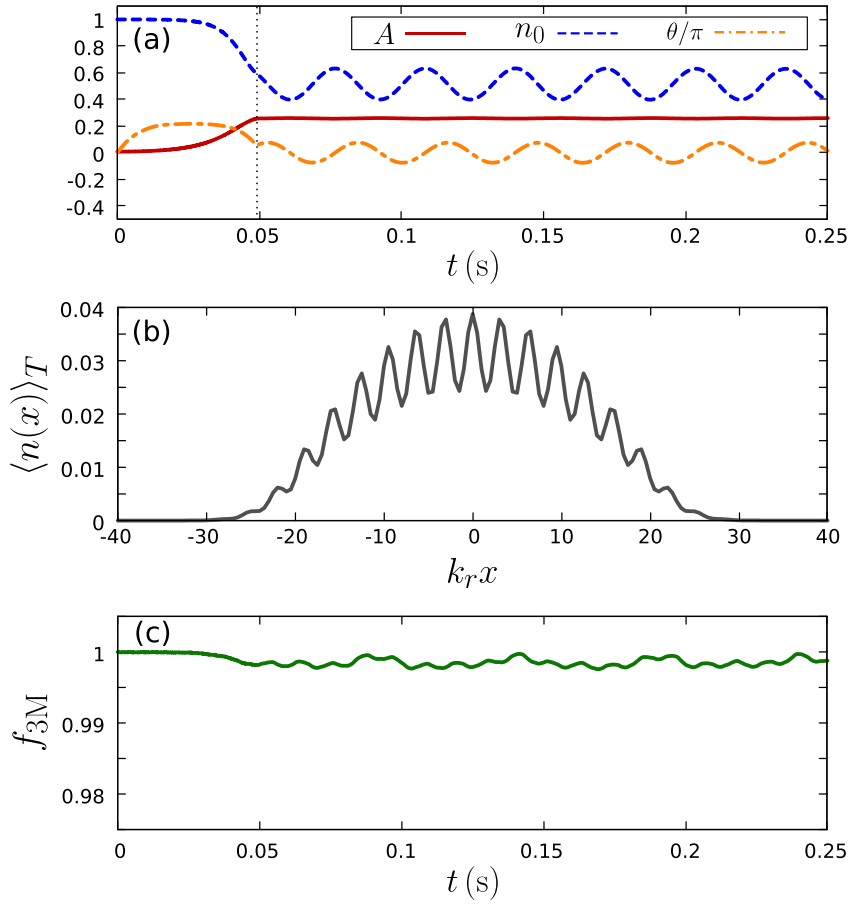

FIG. 7. Excitation of ES states via coherent spin mixing: GPE results. (a) $n_{0}$ (dashed blue), $\theta$ (dotted-dashed orange), and $A(t)$ (solid red) as a function of time for a state initially prepared at $n_{0}=0.9998$ and $\theta=0$. The state is evolved with the GPE (14), for $N=10^{4}, \Omega=0.75 E_{r}$, and $\omega_{\mathrm{t}}$ adjusted to have $\bar{n}=7.5 \times 10^{13} \mathrm{~cm}^{-3}$. For $t \leqslant t_{1}=5.5 \hbar / \lambda$, we set $\tilde{\epsilon} / \lambda=-1$. At $t=t_{1}$ (dotted vertical line) $\tilde{\epsilon}$ is quenched to 0 . (b) Corresponding density profile $\langle n(x)\rangle_{T}$, time averaged from $t=t_{1}$ to $t=0.25 \mathrm{~s}$. (c) Relative occupation of the three-mode subspace $f_{3 \mathrm{M}}$ along the preparation.

patible with the lifetime of spin-1 Raman-dressed BECs in the considered regimes [80]. Furthermore, we consider a $10 \%$ Gaussian uncertainty in $N(0)$. The background magnetic noise is accounted for via sinusoidal modulations of $\delta$ and $\epsilon$ at frequency $50 \mathrm{~Hz}$. We set their amplitudes, respectively, to 700 and $5 \mathrm{~Hz}$, which roughly correspond to a magnetic bias field of $B \sim 35 \mathrm{G}$ with $\sim 1 \mathrm{mG}$ instability in experiments with $F=1{ }^{87} \mathrm{Rb}$ atoms. We consider a Gaussian uncertainty of $\pm 5 \%$ in $\Omega$ to match the systematic uncertainty reported in [72]. Finally, a finite bias field unavoidably results in cross coupling between the two Raman-dressed Zeeman state pairs. This cross coupling is translated into an effective shift in the value of $\epsilon$ that depends on $\Omega$, which can be computed from Floquet theory. We use the polynomial expression for the shift as given in Methods from [72].

With all these considerations, we reproduce the protocol as described in the previous section, incorporating now the uncertainties in the parameters. In Fig. 8(a1) we plot the corresponding mean value and standard deviation of $A(t), n_{0}$, and $f_{3 \mathrm{M}}$ as a function of time, evaluated from a sample of 20 realizations. Despite the addition of noise, the preparation still yields large and stable modulations in the density profile for the parameters chosen. As discussed in the previous section, the tunability of the Raman-mediated spin mixing allows the realization of the protocol in larger condensates. This can be achieved by setting a lower $\Omega$ (see Fig. 4), but at the expense 
(a)

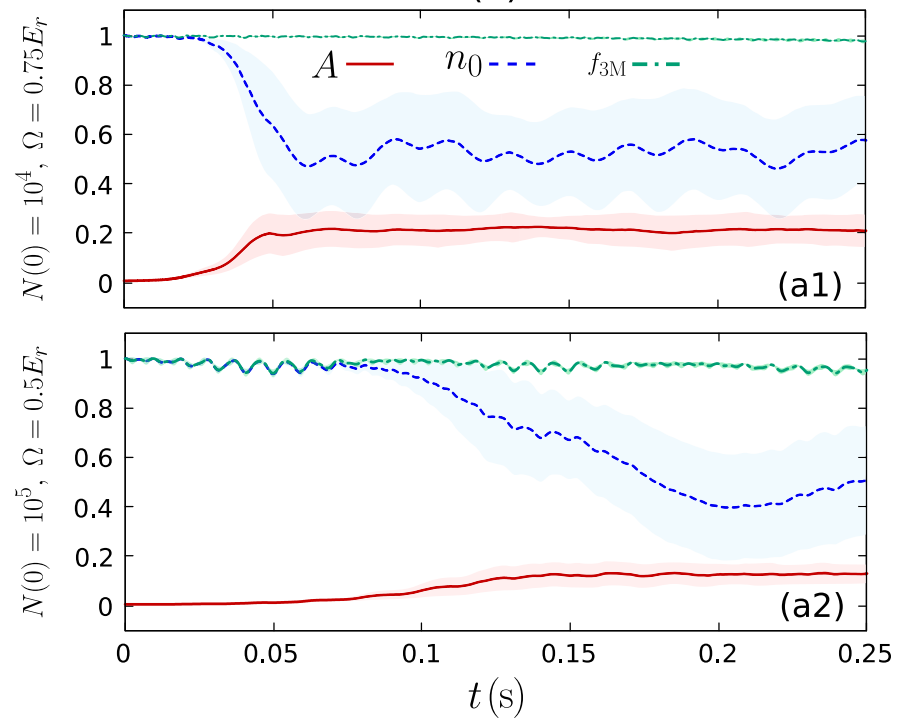

(b)
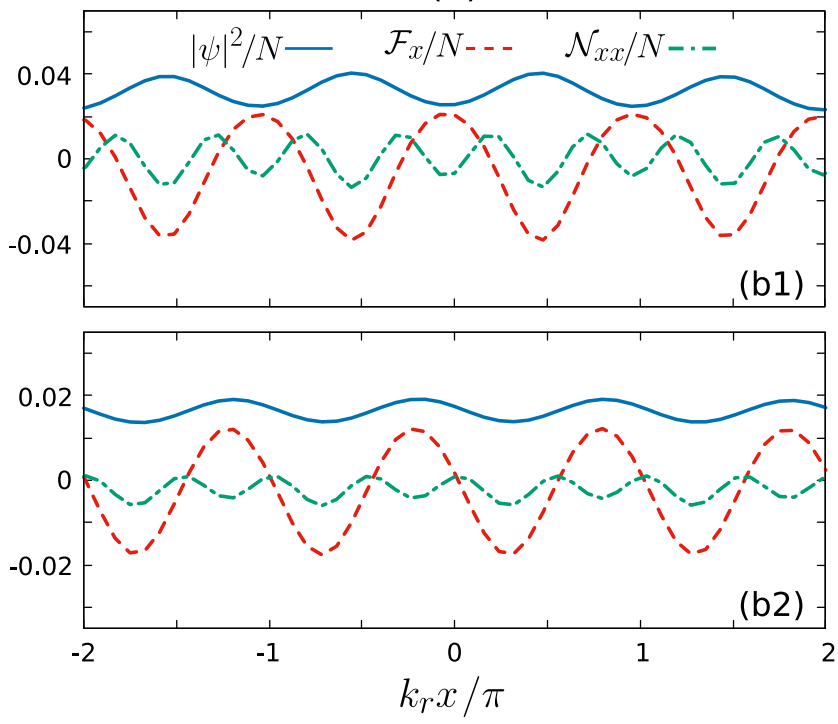

FIG. 8. Robust excitation of ES states. (a) Mean value of $A(t)$ (red solid line), $n_{0}$ (blue dashed line), and $f_{3 \mathrm{M}}$ (green dash-dotted line) as a function of time, for a state with $n_{0}(0)=0.9998$ and $\theta(0)=0$. The state is evolved under the GPE $(14)$, with $N(t)=N(0) \exp (-\gamma t)$ and including randomized parameters to account for atom loss and experimental noise (see main text). In (a1) we set $N(0)=10^{4}$ and $\Omega=0.75 E_{r}$. In (a2) we set $N(0)=10^{5}$ and $\Omega=0.5 E_{r}$. In both cases $\gamma=3.33$, and $\tilde{\epsilon} / \lambda=-1$ for $t \leqslant t_{1}=5.5 \hbar / \lambda$ and $\tilde{\epsilon}=0$ for $t>t_{1}$. The trap frequency $\omega_{\mathrm{t}}$ is adjusted to have $\bar{n}(0)=7.5 \times 10^{13} \mathrm{~cm}^{-3}$. The averages are computed from a sample of 20 realizations, with the light-colored shadowed regions indicating the associated standard deviation. (b) Longitudinal density $|\boldsymbol{\psi}|^{2}$ (blue solid line), spin density $\mathcal{F}_{x}$ (red dashed line), and nematic density $\mathcal{N}_{x x}$ (green dash-dotted line) at $t=t_{1}$, evaluated for a single realization from the samples used in (a).

of a smaller contrast of the stripes, as well as of detrimental effects from noise and atom loss. This is exemplified in Fig. 8(a2), where we plot the results for an analogous preparation with $N(0)=10^{5}$ and $\Omega=0.5 E_{r}$. The trap frequency is adjusted to initially have $\bar{n}=7.5 \times 10^{13} \mathrm{~cm}^{-3}$. While smaller, the amplitude $A(t)$ is stabilized in less than $200 \mathrm{~ms}$, with over half the atoms remaining in the condensate.

In Fig. 8(b) we plot the longitudinal density $\left|\boldsymbol{\psi}^{2}\right|$, the spin density $\mathcal{F}_{x}=\boldsymbol{\psi}^{*} \hat{F}_{x} \boldsymbol{\psi}$, and the nematic density $\mathcal{N}_{x x}=$ $\boldsymbol{\psi}^{*}\left(2 / 3-\hat{F}_{x}^{2}\right) \boldsymbol{\psi}$ at $t=t_{1}$, right after the quench to $\tilde{\epsilon}=0$. The quantities are computed for a randomly chosen realization from the samples used in Fig. 8(a). The values shown are not time averaged since the instability in $\delta$ induces a backand-forth displacement of the stripes. However, as discussed in Sec. III, the width of the stripes remains stable over time, according to (10). In the prepared ES states, the periodicity of the spatial modulations match those of the ground-state ferromagnetic stripe phase [71], with the particle density and the spin densities having periodicity $2 \pi /\left|\boldsymbol{k}_{\mathbf{1}}\right|$, and the nematic densities containing harmonic components both with period $2 \pi /\left|\boldsymbol{k}_{\mathbf{1}}\right|$ and $\pi /\left|\boldsymbol{k}_{\mathbf{1}}\right|$. Remarkably, this preparation of stripe states via crossing an ESQP transition of the effective model compares favorably, both in its robustness and in the contrast achieved, to the quasiadiabatic preparation through a quantum phase transition proposed in [41].

As discussed in Sec. III, due to the instability in the relative phase $\Delta$ between the modes $b_{ \pm 1}$, positive and negative values of $A(t)$ cannot be distinguished experimentally. However, in the states prepared, the contrast of the stripes $V \sim 2|A|$ remains stable over time and does not vanish at any given time, which is the distinct feature of the ES phase. At the same time, this stability provides a direct measurement of the winding number $w$ that characterises the $\mathrm{BA}^{\prime} \mathrm{ESQP}$ of the effective spin Hamiltonian.

\section{CONCLUSIONS}

In this work we have studied the emergence of ESQPs in Raman-dressed SOC spin-1 condensates. Following a dressed-base description, the SOC gas can be interpreted as an undressed spinor gas with effective tunable spin-spin interactions. With this in mind, we have directly connected the corresponding ESQPs of the bare spinor gas to those of the Raman-dressed system. Moreover, due to the coupling between internal (spin) and external (motional) degrees of freedom in the presence of SOC, the phases of the dressed condensate exhibit richer features. Most relevantly, a novel ESQP can be defined in the dressed system, the ES phase, where the atomic cloud exhibits stable density modulations that do not vanish over time. The nature of the phase is understood from the topological order parameter that characterizes the ESQPs of the spinor gas in the regime where the system is described by a collective spin Hamiltonian.

We have numerically assessed the predictions of the effective model with simulations of the GPE of the dressed condensate. We find that, indeed, the collective spin structure Hamiltonian plays a fundamental role to the existence of the ES phase, with its signature quickly vanishing when the few-mode truncation that leads to the effective Hamiltonian is significantly challenged. While such a sensitivity supposes a restriction to its experimental realization, we have shown that the large tunability of the system allows a wide regime of parameters for which the phase is supported. 
At the same time, we have shown that the realization of the spin Hamiltonian in the dressed condensate can be advantageous when it comes to the detection of the ESQP transitions of the system. So far, the proposal to measure the topological order parameter in undressed quantum gases [38] relies on an interferometric protocol that is very sensitive to magnetic field fluctuations. In contrast, in the Raman-dressed gas, the same information can be obtained from direct measurements of the density profile of the atomic cloud, with an order parameter, the minimum contrast of the spatial modulations, that is insensitive to fluctuations of the bias field.

Finally, by exploiting the properties of the ES phase, we have proposed a simple scheme to prepare stripe states with large and stable density modulations. We have numerically tested the robustness of such a preparation with the GPE, and found it to be feasible in state-of-the-art experiments with Raman-dressed spinor condensates.

Our pseudospin description of the Raman-dressed gas across the whole spectrum of the collective spin Hamiltonian suggests alternative directions for achieving macroscopic entanglement in momentum space [81]. The squeezing and quantum correlations generated by our two-step quench scheme can be calculated for a mesoscopic number of particles by including the effect of atoms' losses in the single-mode quantum description by wave-function Monte Carlo [82] as recently done in [68]. Beyond the single-mode approximation, the properties of the quantum correlations in the system could be explored for few particles, for instance in connection to the two-particle solutions of the model, as done in [83] for the spin- $\frac{1}{2}$ scenario.

\section{ACKNOWLEDGMENTS}

We thank L. Tarruell for insightful discussions on experimental aspects of the Raman coupled BEC. We acknowledge support from the Ministerio de Economía y Competitividad MINECO (Contracts No. FIS2017-86530-P and No. PID2020-112687GB-C22), from the European Regional Development Fund within the ERDF Operational Program of Catalunya (project QUASICAT/QuantumCat, Ref. 001-P001644), and from Generalitat de Catalunya (Contract No. SGR2017-1646). A.C. acknowledges support from the Universitat Autònoma de Barcelona Talent Research program.
[1] C. Gardiner and P. Zoller, The Quantum World of Ultra-Cold Atoms and Light Book II: The Physics of Quantum-Optical Devices (Imperial College Press, London, 2015).

[2] A. Celi, A. Sanpera, V. Ahufinger, and M. Lewenstein, Quantum optics and frontiers of physics: The third quantum revolution, Phys. Scr. 92, 013003 (2017).

[3] J. Dalibard, F. Gerbier, G. Juzeliūnas, and P. Öhberg, Colloquium: Artificial gauge potentials for neutral atoms, Rev. Mod. Phys. 83, 1523 (2011).

[4] N. Goldman, G. Juzeliūnas, P. Öhberg, and I. B. Spielman, Light-induced gauge fields for ultracold atoms, Rep. Prog. Phys. 77, 126401 (2014).

[5] M. Lewenstein, A. Sanpera, and V. Ahufinger, Ultracold Atoms in Optical Lattices: Simulating Quantum Many-Body Systems (Oxford University Press, Oxford, 2012).

[6] Y.-J. Lin, R. L. Compton, K. Jiménez-García, J. V. Porto, and I. B. Spielman, Synthetic magnetic fields for ultracold neutral atoms, Nature (London) 462, 628 (2009).

[7] Y.-J. Lin, K. Jiménez-García, and I. B. Spielman, Spin-orbitcoupled Bose-Einstein condensates, Nature (London) 471, 83 (2011).

[8] R. A. Williams, L. J. LeBlanc, K. Jimenez-Garcia, M. C. Beeler, A. R. Perry, W. D. Phillips, and I. B. Spielman, Synthetic partial waves in ultracold atomic collisions, Science 335, 314 (2012).

[9] J.-R. Li, J. Lee, W. Huang, S. Burchesky, B. Shteynas, F. Ç. Top, A. O. Jamison, and W. Ketterle, A stripe phase with supersolid properties in spin-orbit-coupled Bose-Einstein condensates, Nature (London) 543, 91 (2017).

[10] A. Putra, F. Salces-Cárcoba, Y. Yue, S. Sugawa, and I. B. Spielman, Spatial Coherence of Spin-Orbit-Coupled Bose Gases, Phys. Rev. Lett. 124, 053605 (2020).

[11] J. Hou, X.-W. Luo, K. Sun, T. Bersano, V. Gokhroo, S. Mossman, P. Engels, and C. Zhang, Momentum-Space Josephson Effects, Phys. Rev. Lett. 120, 120401 (2018).
[12] L. Tanzi, E. Lucioni, F. Famà, J. Catani, A. Fioretti, C. Gabbanini, R. N. Bisset, L. Santos, and G. Modugno, Observation of a Dipolar Quantum Gas with Metastable Supersolid Properties, Phys. Rev. Lett. 122, 130405 (2019).

[13] F. Böttcher, J.-N. Schmidt, M. Wenzel, J. Hertkorn, M. Guo, T. Langen, and T. Pfau, Transient Supersolid Properties in an Array of Dipolar Quantum Droplets, Phys. Rev. X 9, 011051 (2019).

[14] L. Chomaz, D. Petter, P. Ilzhöfer, G. Natale, A. Trautmann, C. Politi, G. Durastante, R. M. W. van Bijnen, A. Patscheider, M. Sohmen, M. J. Mark, and F. Ferlaino, Long-Lived and Transient Supersolid Behaviors in Dipolar Quantum Gases, Phys. Rev. X 9, 021012 (2019).

[15] R. Ramos, A. Frölian, C. Chisholm, E. Neri, C. Cabrera, A. Celi, and L. Tarruell, Realization of a chiral BF theory in an optically dressed Bose-Einstein condensate, Bull. Am. Phys. Soc. Z06, Z06.00006 (2021)

[16] M. Heyl, Dynamical quantum phase transitions: A review, Rep. Prog. Phys. 81, 054001 (2018).

[17] P. Cejnar, P. Stránský, M. Macek, and M. Kloc, Excited-state quantum phase transitions, J. Phys. A: Math. Theor. 54, 133001 (2021).

[18] M. Vojta, Quantum phase transitions, Rep. Prog. Phys. 66, 2069 (2003).

[19] S. Sachdev, Quantum Phase Transitions, 2nd ed. (Cambridge University Press, Cambridge, England, 2011).

[20] N. Fläschner, D. Vogel, M. Tarnowski, B. S. Rem, D.-S. Lühmann, M. Heyl, J. C. Budich, L. Mathey, K. Sengstock, and C. Weitenberg, Observation of dynamical vortices after quenches in a system with topology, Nat. Phys. 14, 265 (2018).

[21] W. Sun, C.-R. Yi, B.-Z. Wang, W.-W. Zhang, B. C. Sanders, X.-T. Xu, Z.-Y. Wang, J. Schmiedmayer, Y. Deng, X.-J. Liu, S. Chen, and J.-W. Pan, Uncover Topology by Quantum Quench Dynamics, Phys. Rev. Lett. 121, 250403 (2018). 
[22] S. Smale, P. He, B. A. Olsen, K. G. Jackson, H. Sharum, S. Trotzky, J. Marino, A. M. Rey, and J. H. Thywissen, Observation of a transition between dynamical phases in a quantum degenerate Fermi gas, Sci. Adv. 5, eaax1568 (2019).

[23] J. A. Muniz, D. Barberena, R. J. Lewis-Swan, D. J. Young, J. R. K. Cline, A. M. Rey, and J. K. Thompson, Exploring dynamical phase transitions with cold atoms in an optical cavity, Nature (London) 580, 602 (2020).

[24] P. Jurcevic, H. Shen, P. Hauke, C. Maier, T. Brydges, C. Hempel, B. P. Lanyon, M. Heyl, R. Blatt, and C. F. Roos, Direct Observation of Dynamical Quantum Phase Transitions in an Interacting Many-Body System, Phys. Rev. Lett. 119, 080501 (2017).

[25] J. Zhang, G. Pagano, P. W. Hess, A. Kyprianidis, P. Becker, H. Kaplan, A. V. Gorshkov, Z.-X. Gong, and C. Monroe, Observation of a many-body dynamical phase transition with a 53-qubit quantum simulator, Nature (London) 551, 601 (2017).

[26] K. Xu, Z.-H. Sun, W. Liu, Y.-R. Zhang, H. Li, H. Dong, W. Ren, P. Zhang, F. Nori, D. Zheng, H. Fan, and H. Wang, Probing dynamical phase transitions with a superconducting quantum simulator, Sci. Adv. 6, eaba4935 (2020).

[27] F. Leyvraz and W. D. Heiss, Large- $n$ Scaling Behavior of the Lipkin-Meshkov-Glick Model, Phys. Rev. Lett. 95, 050402 (2005).

[28] P. Ribeiro, J. Vidal, and R. Mosseri, Thermodynamical Limit of the Lipkin-Meshkov-Glick Model, Phys. Rev. Lett. 99, 050402 (2007).

[29] M. Caprio, P. Cejnar, and F. Iachello, Excited state quantum phase transitions in many-body systems, Ann. Phys. (NY) 323, 1106 (2008).

[30] D. Larese, F. Pérez-Bernal, and F. Iachello, Signatures of quantum phase transitions and excited state quantum phase transitions in the vibrational bending dynamics of triatomic molecules, J. Mol. Struct. 1051, 310 (2013).

[31] T. Brandes, Excited-state quantum phase transitions in Dicke superradiance models, Phys. Rev. E 88, 032133 (2013).

[32] P. Stránský, M. Macek, and P. Cejnar, Excited-state quantum phase transitions in systems with two degrees of freedom: Level density, level dynamics, thermal properties, Ann. Phys. 345, 73 (2014).

[33] L. F. Santos, M. Távora, and F. Pérez-Bernal, Excited-state quantum phase transitions in many-body systems with infiniterange interaction: Localization, dynamics, and bifurcation, Phys. Rev. A 94, 012113 (2016).

[34] T. Opatrný, L. Richterek, and M. Opatrný, Analogies of the classical Euler top with a rotor to spin squeezing and quantum phase transitions in a generalized Lipkin-Meshkov-Glick model, Sci. Rep. 8, 1984 (2018).

[35] M. Macek, P. Stránský, A. Leviatan, and P. Cejnar, Excitedstate quantum phase transitions in systems with two degrees of freedom. III. Interacting boson systems, Phys. Rev. C 99, 064323 (2019).

[36] B. Dietz, F. Iachello, M. Miski-Oglu, N. Pietralla, A. Richter, L. von Smekal, and J. Wambach, Lifshitz and excited-state quantum phase transitions in microwave Dirac billiards, Phys. Rev. B 88, 104101 (2013).

[37] C. B. Dağ, S.-T. Wang, and L.-M. Duan, Classification of quench-dynamical behaviors in spinor condensates, Phys. Rev. A 97, 023603 (2018).
[38] P. Feldmann, C. Klempt, A. Smerzi, L. Santos, and M. Gessner, Interferometric Order Parameter for Excited-State Quantum Phase Transitions in Bose-Einstein Condensates, Phys. Rev. Lett. 126, 230602 (2021).

[39] H.-X. Yang, T. Tian, Y.-B. Yang, L.-Y. Qiu, H.-Y. Liang, A.-J. Chu, C. B. Dağ, Y. Xu, Y. Liu, and L.-M. Duan, Observation of dynamical quantum phase transitions in a spinor condensate, Phys. Rev. A 100, 013622 (2019).

[40] T. Tian, H.-X. Yang, L.-Y. Qiu, H.-Y. Liang, Y.-B. Yang, Y. Xu, and L.-M. Duan, Observation of Dynamical Quantum Phase Transitions with Correspondence in an Excited State Phase Diagram, Phys. Rev. Lett. 124, 043001 (2020).

[41] J. Cabedo, J. Claramunt, and A. Celi, Dynamical preparation of stripe states in spin-orbit-coupled gases, Phys. Rev. A 104, L031305 (2021).

[42] M. Kitagawa and M. Ueda, Squeezed spin states, Phys. Rev. A 47, 5138 (1993).

[43] C. K. Law, H. Pu, and N. P. Bigelow, Quantum Spins Mixing in Spinor Bose-Einstein Condensates, Phys. Rev. Lett. 81, 5257 (1998).

[44] L.-M. Duan, A. Sørensen, J. I. Cirac, and P. Zoller, Squeezing and Entanglement of Atomic Beams, Phys. Rev. Lett. 85, 3991 (2000).

[45] D. M. Stamper-Kurn and M. Ueda, Spinor Bose gases: Symmetries, magnetism, and quantum dynamics, Rev. Mod. Phys. 85 1191 (2013).

[46] J. Stenger, S. Inouye, D. M. Stamper-Kurn, H.-J. Miesner, A. P. Chikkatur, and W. Ketterle, Spin domains in ground-state BoseEinstein condensates, Nature (London) 396, 345 (1998).

[47] L. E. Sadler, J. M. Higbie, S. R. Leslie, M. Vengalattore, and D. M. Stamper-Kurn, Spontaneous symmetry breaking in a quenched ferromagnetic spinor Bose-Einstein condensate, Nature (London) 443, 312 (2006).

[48] E. M. Bookjans, A. Vinit, and C. Raman, Quantum Phase Transition in an Antiferromagnetic Spinor Bose-Einstein Condensate, Phys. Rev. Lett. 107, 195306 (2011).

[49] A. Vinit, E. M. Bookjans, C. A. R. Sa de Melo, and C. Raman, Antiferromagnetic Spatial Ordering in a Quenched One-Dimensional Spinor Gas, Phys. Rev. Lett. 110, 165301 (2013).

[50] T. M. Hoang, M. Anquez, B. A. Robbins, X. Y. Yang, B. J. Land, C. D. Hamley, and M. S. Chapman, Parametric excitation and squeezing in a many-body spinor condensate, Nat. Commun. 7, 11233 (2016).

[51] M. Anquez, B. A. Robbins, H. M. Bharath, M. Boguslawski, T. M. Hoang, and M. S. Chapman, Quantum Kibble-Zurek Mechanism in a Spin-1 Bose-Einstein Condensate, Phys. Rev. Lett. 116, 155301 (2016).

[52] M. Prüfer, P. Kunkel, H. Strobel, S. Lannig, D. Linnemann, C.-M. Schmied, J. Berges, T. Gasenzer, and M. K. Oberthaler, Observation of universal dynamics in a spinor Bose gas far from equilibrium, Nature (London) 563, 217 (2018).

[53] Z. Chen, T. Tang, J. Austin, Z. Shaw, L. Zhao, and Y. Liu, Quantum Quench and Nonequilibrium Dynamics in Lattice-Confined Spinor Condensates, Phys. Rev. Lett. 123, 113002 (2019).

[54] S. Kang, S. W. Seo, H. Takeuchi, and Y. Shin, Observation of Wall-Vortex Composite Defects in a Spinor Bose-Einstein Condensate, Phys. Rev. Lett. 122, 095301 (2019).

[55] K. Jiménez-García, A. Invernizzi, B. Evrard, C. Frapolli, J. Dalibard, and F. Gerbier, Spontaneous formation and relaxation 
of spin domains in antiferromagnetic spin-1 condensates, Nat. Commun. 10, 1422 (2019).

[56] M. Prüfer, T. V. Zache, P. Kunkel, S. Lannig, A. Bonnin, H. Strobel, J. Berges, and M. K. Oberthaler, Experimental extraction of the quantum effective action for a non-equilibrium many-body system, Nat. Phys. 16, 1012 (2020).

[57] E. M. Bookjans, C. D. Hamley, and M. S. Chapman, Strong Quantum Spin Correlations Observed in Atomic Spin Mixing, Phys. Rev. Lett. 107, 210406 (2011).

[58] B. Lücke, M. Scherer, J. Kruse, L. Pezzé, F. Deuretzbacher, P. Hyllus, O. Topic, J. Peise, W. Ertmer, J. Arlt, L. Santos, A. Smerzi, and C. Klempt, Twin matter waves for interferometry beyond the classical limit, Science 334, 773 (2011).

[59] C. Gross, H. Strobel, E. Nicklas, T. Zibold, N. Bar-Gill, G. Kurizki, and M. K. Oberthaler, Atomic homodyne detection of continuous-variable entangled twin-atom states, Nature (London) 480, 219 (2011).

[60] C. D. Hamley, C. S. Gerving, T. M. Hoang, E. M. Bookjans, and M. S. Chapman, Spin-nematic squeezed vacuum in a quantum gas, Nat. Phys. 8, 305 (2012).

[61] Z. Zhang and L.-M. Duan, Generation of Massive Entanglement through an Adiabatic Quantum Phase Transition in a Spinor Condensate, Phys. Rev. Lett. 111, 180401 (2013).

[62] M. Gabbrielli, L. Pezzè, and A. Smerzi, Spin-Mixing Interferometry with Bose-Einstein Condensates, Phys. Rev. Lett. 115, 163002 (2015).

[63] I. Peise, J.and Kruse, K. Lange, B. Lücke, L. Pezzè, J. Arlt, W. Ertmer, K. Hammerer, L. Santos, A. Smerzi, and C. Klempt, Satisfying the Einstein-Podolsky-Rosen criterion with massive particles, Nat. Commun. 6, 8984 (2015).

[64] T. M. Hoang, H. M. Bharath, M. J. Boguslawski, M. Anquez, B. A. Robbins, and M. S. Chapman, Adiabatic quenches and characterization of amplitude excitations in a continuous quantum phase transition, Proc. Natl. Acad. Sci. USA 113, 9475 (2016).

[65] X.-Y. Luo, Y.-Q. Zou, L.-N. Wu, Q. Liu, M.-F. Han, M. K. Tey, and L. You, Deterministic entanglement generation from driving through quantum phase transitions, Science 355, 620 (2017).

[66] Y.-Q. Zou, L.-N. Wu, Q. Liu, X.-Y. Luo, S.-F. Guo, J.-H. Cao, M. K. Tey, and L. You, Beating the classical precision limit with spin-1 Dicke states of more than 10,000 atoms, Proc. Natl. Acad. Sci. USA 115, 6381 (2018).

[67] P. Kunkel, M. Prüfer, H. Strobel, D. Linnemann, A. Frölian, T. Gasenzer, M. Gärttner, and M. K. Oberthaler, Spatially distributed multipartite entanglement enables EPR steering of atomic clouds, Science 360, 413 (2018).

[68] L. Pezzè, M. Gessner, P. Feldmann, C. Klempt, L. Santos, and A. Smerzi, Heralded Generation of Macroscopic Superposition States in a Spinor Bose-Einstein Condensate, Phys. Rev. Lett. 123, 260403 (2019).
[69] A. Qu, B. Evrard, J. Dalibard, and F. Gerbier, Probing Spin Correlations in a Bose-Einstein Condensate Near the SingleAtom Level, Phys. Rev. Lett. 125, 033401 (2020).

[70] L. Pezzè, A. Smerzi, M. K. Oberthaler, R. Schmied, and P. Treutlein, Quantum metrology with nonclassical states of atomic ensembles, Rev. Mod. Phys. 90, 035005 (2018).

[71] G. I. Martone, F. V. Pepe, P. Facchi, S. Pascazio, and S. Stringari, Tricriticalities and Quantum Phases in Spin-OrbitCoupled Spin-1 Bose Gases, Phys. Rev. Lett. 117, 125301 (2016).

[72] D. L. Campbell, R. M. Price, A. Putra, A. Valdés-Curiel, D. Trypogeorgos, and I. B. Spielman, Magnetic phases of spin-1 spin-orbit-coupled Bose gases, Nat. Commun. 7, 10897 (2016).

[73] K. Murata, H. Saito, and M. Ueda, Broken-axisymmetry phase of a spin-1 ferromagnetic Bose-Einstein condensate, Phys. Rev. A 75, 013607 (2007).

[74] A. L. Corps and A. Relaño, Constant of Motion Identifying Excited-State Quantum Phases, Phys. Rev. Lett. 127, 130602 (2021).

[75] W. Zhang, D. L. Zhou, M.-S. Chang, M. S. Chapman, and L. You, Coherent spin mixing dynamics in a spin-1 atomic condensate, Phys. Rev. A 72, 013602 (2005).

[76] Y. Kawaguchi and M. Ueda, Spinor Bose-Einstein condensates, Phys. Rep. 520, 253 (2012).

[77] S. Yi, O. E. Müstecaplığlu, C. P. Sun, and L. You, Single-mode approximation in a spinor-1 atomic condensate, Phys. Rev. A 66, 011601(R) (2002).

[78] C. Klempt, O. Topic, G. Gebreyesus, M. Scherer, T. Henninger, P. Hyllus, W. Ertmer, L. Santos, and J. J. Arlt, Parametric Amplification of Vacuum Fluctuations in a Spinor Condensate, Phys. Rev. Lett. 104, 195303 (2010).

[79] B. Evrard, A. Qu, J. Dalibard, and F. Gerbier, Coherent seeding of the dynamics of a spinor Bose-Einstein condensate: From quantum to classical behavior, Phys. Rev. A 103, L031302 (2021).

[80] R. P. Anderson, D. Trypogeorgos, A. Valdés-Curiel, Q.-Y. Liang, J. Tao, M. Zhao, T. Andrijauskas, G. Juzeliūnas, and I. B. Spielman, Realization of a deeply subwavelength adiabatic optical lattice, Phys. Rev. Res. 2, 013149 (2020).

[81] F. Anders, A. Idel, P. Feldmann, D. Bondarenko, S. Loriani, K. Lange, J. Peise, M. Gersemann, B. Meyer-Hoppe, S. Abend, N. Gaaloul, C. Schubert, D. Schlippert, L. Santos, E. Rasel, and C. Klempt, Momentum Entanglement for Atom Interferometry, Phys. Rev. Lett. 127, 140402 (2021).

[82] K. Mølmer, Y. Castin, and J. Dalibard, Monte Carlo wavefunction method in quantum optics, J. Opt. Soc. Am. B 10, 524 (1993).

[83] C. Zhu, L. Dong, and H. Pu, Harmonically trapped atoms with spin-orbit coupling, J. Phys. B At. Mol. Opt. Phys. 49, 145301 (2016). 